\title{
A Novel Analytical Method to Calculate Wheel-Rail Tangential Forces and Validation on a Scaled Roller-Rig
}

\author{
Nicola Bosso (i) and Nicolò Zampieri (i) \\ Politecnico di Torino, Department of Mechanical and Aerospace Engineering, C.so Duca degli Abruzzi 24, 10129 Turin, Italy \\ Correspondence should be addressed to Nicola Bosso; nicola.bosso@polito.it
}

Received 30 January 2018; Revised 25 June 2018; Accepted 12 July 2018; Published 9 August 2018

Academic Editor: Huseyin Çimenoğlu

Copyright (c) 2018 Nicola Bosso and Nicolò Zampieri. This is an open access article distributed under the Creative Commons Attribution License, which permits unrestricted use, distribution, and reproduction in any medium, provided the original work is properly cited.

\begin{abstract}
The study of railway dynamic strongly depends on the estimation of the tangential forces acting between wheel and rail. Simulation of the dynamical behaviour of railway vehicles is often performed using multibody codes, and the calculation of the contact forces must be efficient and accurate, even if the contact problem is strongly nonlinear. Therefore, the contact problem is still of great interest for researchers. This work proposes an analytical and efficient algorithm to calculate wheel-rail tangential forces. The proposed method is compared with the most commonly used algorithms under different conditions. In addition, experimental tests are performed on a scaled prototype on roller-rig to demonstrate that the method can be easily adjusted using experimental results. The benefit of the proposed method is to provide an analytical and fast solution, able to obtain accurate results and to allow corrections based on empirical evidence.
\end{abstract}

\section{Introduction}

The calculation of the tangential forces between wheel and rail is a fundamental aspect when studying railway vehicle dynamic. It plays an important role both in the case of steady state running of the vehicle and during traction or braking operations. The determination of the friction forces is a complex problem, as it involves several subproblems that lead to a strong nonlinearity on the behaviour of tangential forces, which are also affected by external conditions (contact contamination).

The problem can be solved accurately using precise but complex algorithms, which require huge computational time [1-3]. Since the problem governs vehicle dynamic, the simulation of the dynamic behaviour of railway vehicles, usually performed by means of multibody codes [4, 5], requires simpler and more efficient algorithms. However, the accuracy of the results should be preserved as much as possible. For this reason, during the last years researchers have developed several numerical methods able to solve the problem efficiently [6-12]. At first, linear models were used to study tangential forces as a function of the relative velocity between wheel and rail, which was briefly described using the kinematical creepages [2]. Later, simple nonlinear laws were proposed in order to take into account the adhesion limit at the contact, such as the hyperbolic tangent method or other heuristic nonlinear approaches based on experimental observations [8]. Kalker, after developing his complete theory, described by complex algorithms, such as CONTACT and DUVROL [2], realized that a simpler method was fundamental for vehicle simulation purposes and studied a simpler but faster method known as FASTSIM [6].

Later, Polach studied an alternative method [7], able to be faster than FASTSIM and provide similar results, that was also compared with experimental tests performed on real vehicles. Polach, in addition, developed an extension of his method in order to investigate adhesion in nonsteady condition related to traction, also considering different environmental conditions, by modifying his original method to include these aspects [13]. Also the algorithms developed by Kalker have been recently improved by Vollebregt, by increasing the accuracy of FASTSIM in the FASTSIM2 version [14] and implementing in the more complex CONTACT code the effect of "falling friction" versus the creepage $[15,16]$, which was already introduced by Polach in his extended method $[13,17]$. Nowadays FASTSIM and Polach's methods are commonly used in multibody codes, to investigate railway vehicle dynamic. The authors of this paper 
had previously proposed a simplified nonlinear method to estimate wheel-rail and wheel-roller tangential forces. That method did not consider accurately the effect of the spin creepage. The present work proposes an improvement of the previously proposed method, which correctly takes into account the spin creepage and allows considering the variation of the friction coefficient with the creepage ("falling friction"), allowing its use in traction/braking simulations. The benefit of the proposed algorithm is that it consists of two analytical equations, for the lateral and longitudinal force, written as a direct function of the three kinematical creepages. The algorithm can be easily adjusted using a set of coefficients to meet the evidence of experimental tests.

\section{Methods}

This work proposes a new algorithm to calculate the wheelrail tangential forces, whose peculiarity is to allow faster calculations and the possibility of improving the results, by modifying the coefficients of the method on the basis of the experimental results. This is possible because the algorithm is formulated in order to be able to modify the shape of the force-creepage behaviour acting on few coefficients.
In order to validate the algorithm, the numerical results have been compared with the most used methods (FASTSIM and Polach) considering at first simple cases and then the simulation of a vehicle on real track. Finally the method is also compared with experimental results obtained on a scaled prototype on roller-rig.

2.1. Tangential Force Calculation. The proposed method for calculating the tangential forces has been improved with respect to a previous method proposed by the authors [10], where the spin creepage was not adequately considered. The new method has corrected the influence of the spin creepage in order to be comparable with the results achieved by the most common algorithms $[6,7]$. This method allows calculating with two analytical equations the longitudinal and lateral force in the contact area according to (1) and (2), as a function of the longitudinal $(\xi)$, lateral $(\eta)$, and spin $(\phi)$ creepages. The creepages are defined in general as the ratio of the relative velocity between wheel and rail in the considered direction and the vehicle velocity $[1,2]$. For the specific case of a wheelset on a roller-rig, adopted in this work for the experimental tests, the expressions of the creepages are slightly different with respect to the case of the wheelset on the rail. Those expressions will be described later ((13)-(17)).

$$
\begin{aligned}
& F_{x}=\frac{f_{11} \cdot \xi}{\sqrt[n]{d+\left(\left(f_{11} \cdot \xi\right) /(\mu \cdot N)\right)^{n_{1}}+\left(\left(f_{22} \cdot \eta\right) /(\mu \cdot N)\right)^{n_{1}}+\left(f_{23} /(\mu \cdot N) \cdot\left(\delta \cdot \phi+k \cdot(\delta \cdot \phi)^{n_{2}}\right)\right)^{n_{1}}}} \\
& F_{y}=\frac{f_{22} \cdot \eta+f_{23} \cdot \phi}{\sqrt[n]{d+\left(\left(f_{11} \cdot \xi\right) /(\mu \cdot N)\right)^{n_{1}}+\left(\left(f_{22} \cdot \eta\right) /(\mu \cdot N)\right)^{n_{1}}+\left(f_{23} /(\mu \cdot N) \cdot\left(\delta \cdot \phi+k \cdot(\delta \cdot \phi)^{n_{2}}\right)\right)^{n_{1}}}}
\end{aligned}
$$

The coefficients $f_{i j}$ can be calculated depending on the dimension of the contact area, considered as elliptical, according to the linear theory of Kalker [2]:

$$
\begin{aligned}
& f_{11}=C_{11} \cdot G \cdot a \cdot b \\
& f_{22}=C_{22} \cdot G \cdot a \cdot b \\
& f_{23}=C_{23} \cdot G \cdot(a \cdot b)^{3 / 2}
\end{aligned}
$$

where $a, b$ are the semiaxes of the contact ellipse and $C_{i j}$ are the so called Kalker's coefficients, which depend on the $a / b$ ratio and on the Poisson's modulus of the material. $G$ is the shear modulus of elasticity.

The $d$ coefficient is usually set equal to 1 and $n=n_{1}$; therefore, when two of the creepages vanish, the formula leads to the Kalker's linear law for small values of the remaining creepage, while tending to $\mu \cdot N$ for high values of the creepage, according to (6) and (7).

The case with pure spin creepage is an exception to the described behaviour, as the lateral force tends to zero for high values of the creepage, according to (8). For low values of the spin creepage, the Kalker's linear law is respected also in this case.

$$
\begin{gathered}
\eta=\phi=0 \Rightarrow F_{x}=\frac{f_{11} \cdot \xi}{\sqrt[n]{1+\left(\left(f_{11} \cdot \xi\right) /(\mu \cdot N)\right)^{n 1}}} \quad \begin{array}{ll}
F_{x}=f_{11} \cdot \xi & \text { for } \xi \longrightarrow 0 \\
F_{x}=\mu \cdot N & \text { for } \xi \longrightarrow \infty
\end{array} \\
\xi=\phi=0 \Rightarrow F_{y}=\frac{f_{22} \cdot \eta}{\sqrt[n]{1+\left(\left(f_{22} \cdot \eta\right) /(\mu \cdot N)\right)^{n 1}}} \quad \begin{cases}F_{y}=f_{22} \cdot \eta & \text { for } \eta \longrightarrow 0 \\
F_{y}=\mu \cdot N & \text { for } \eta \longrightarrow \infty\end{cases} \\
\xi=\eta=0 \Rightarrow F_{y}=\frac{f_{23} \cdot \phi}{\sqrt[n]{1+\left(f_{23} /(\mu \cdot N) \cdot\left(\delta \cdot \phi+k \cdot(\delta \cdot \phi)^{n_{2}}\right)\right)^{n 1}}} \quad \begin{cases}F_{y}=f_{23} \cdot \phi & \text { for } \phi \longrightarrow 0 \\
F_{y}=0 & \text { for } \phi \longrightarrow \infty\end{cases}
\end{gathered}
$$


TABLE 1: Exponents used in the proposed method.

\begin{tabular}{lcccccc}
\hline$n$ & $n_{1}$ & $n_{2}$ & $n_{3}$ & $n_{4}$ & $n_{5}$ & \\
\hline 2.4 & 2.4 & 3.83 & 0.383 & $4 \cdot \pi / 3$ & 2.8 & \\
\hline
\end{tabular}

All the parameters, as the normal force $(N)$, the friction coefficient $(\mu)$, and the longitudinal, lateral, and spin creepages can be calculated according to the dynamic of the wheelset. The $n$ and $n_{1}$ exponents are the most relevant and can be used to modify the shape of the curve and to fit the experimental data. The benefits of the method are that a single analytical equation can be used to calculate the tangential forces, with a very low computational time and that the relevant coefficients can be corrected on the basis of the experimental results.

To define the effect of the spin creepage, in the denominator of the equations, two additional terms must be calculated. The first term $\delta$, according to (9), depends on the $a / b$ ratio and on the exponent $n_{3}$.

$$
\delta=\frac{1}{2} \cdot\left(\frac{b}{a}\right)^{n_{3}}
$$

The second term $k$ is calculated according to (10), depending on the tangential modulus of elasticity, the product of the ellipse semiaxis, the normal load $N$, and the friction coefficient $\mu$, considering three different exponents, $n, n_{4}$, and $n_{5}$.

$$
k=4000 \cdot G^{n} \cdot \frac{(a \cdot b)^{n_{4}}}{(\mu \cdot N)^{n_{5}}}
$$

The exponents adopted on the method for the simulation of Sections 3.1 and 3.2 are shown in Table 1. These exponents are obtained using a trial and error approach in order to achieve, with the proposed algorithm, results similar to the FASTSIM [6] and Polach's [7] algorithms.

The adoption of the exponents of Table 1 and of a constant friction coefficient $\mu=\mu_{0}$ allows describing the dynamic of a vehicle in steady state conditions. This configuration is usually adopted to study dynamic problems, such as the vehicle running stability and the safety against derailment (when the vehicle is running on curves). Instead, when it is necessary to study the behaviour of the contact forces in nonsteady condition, e.g., under an intense and continuous traction effort, the adhesion occurring at the wheel-rail contact decreases versus the value of the creepage ("falling friction" phenomenon).

This variation of the adhesion is usually taken into account in the other methods [13,15-17] by considering a friction coefficient $\mu$, variable as a function of the vehicle velocity, creepage, or other parameters (environmental conditions, track contamination).

This strategy can be used also with the proposed method, by modifying the value of $\mu$ and maintaining the other parameters of the method as indicated in Table 1.

Alternatively, the proposed method also allows a simper strategy to simulate the effect of the falling friction versus the creepage. It consists in the adoption of an exponent $n_{1}>n$
TABLE 2: Technical data of the single unit motor coach Aln663.

\begin{tabular}{lc}
\hline Total mass & $40 \mathrm{t}$ \\
Axle load & $10 \mathrm{t} / \mathrm{axl}$ \\
Wheelbase & $2450 \mathrm{~mm}$ \\
Bogie centre distance & $15940 \mathrm{~mm}$ \\
Wheel diameter & $920 \mathrm{~mm}$ \\
Track gauge & $1435 \mathrm{~mm}$ \\
Wheel arrangement & $(1 \mathrm{~A})(\mathrm{A} 1)$ \\
Maximum speed & $130 \mathrm{~km} / \mathrm{h}$ \\
Power & $2 \times 200 \mathrm{~kW} 1.850 \mathrm{rpm}$ \\
\hline
\end{tabular}

(a reduction of $n_{1}$ from $2 \%$ to $15 \%$ reproduces the typical experimental behaviour). An example of the application of this procedure is shown in Figure 3.

In this case $\left(n_{1} \neq n\right)$, the coefficient $d$ must be modified according to (11) in order to maintain the same initial slope of the curve, while the friction coefficient can be eventually modified according to (12), to keep the same maximum value of the maximum adhesion (with respect to the case with $n_{1}=$ $n)$.

$$
\begin{aligned}
& d=1+n_{1}-n \\
& \mu=\mu_{0} \cdot\left(1+0.6 \cdot\left(n-n_{1}\right)\right)^{n_{6}}
\end{aligned}
$$

2.2. Vehicle Model. The algorithm to calculate the wheel/rail tangential forces proposed in this work is tested in Section 3.2, by simulating a full vehicle running on the track. The numerical model of the vehicle, developed in Simpack 2017, considers a single Diesel Railcar Class Aln663, developed by FIAT Ferroviaria at the beginning of the 80s, running on the Aosta-Pré-Saint-Didier track.

The model is realized considering 17 rigid bodies: one coach frame, 2 bogie frames, 2 bolsters, 4 wheelsets, and 8 axle-boxes. Elastic elements are used to connect the bodies, except the axle-boxes, which are connected to the corresponding wheelsets using rigid revolute joints. The more important data of interest of the vehicle are shown in Table 2.

The Simpack equivalent elastic contact model is used to evaluate the contact point position and the normal load, while the friction forces are evaluated comparing three different algorithms: FASTSIM (considering a discretization of the contact area equal to 50x50), Polach, and the one proposed in this work. The first two algorithms were already included in the Simpack library, while the other one has been added by the authors in the multibody software by means of an external user routine developed in Fortran language. In terms of performances or handling there is no difference between Simpack standard modeling elements and user routines since they are treated the same way. The user routines are in fact 


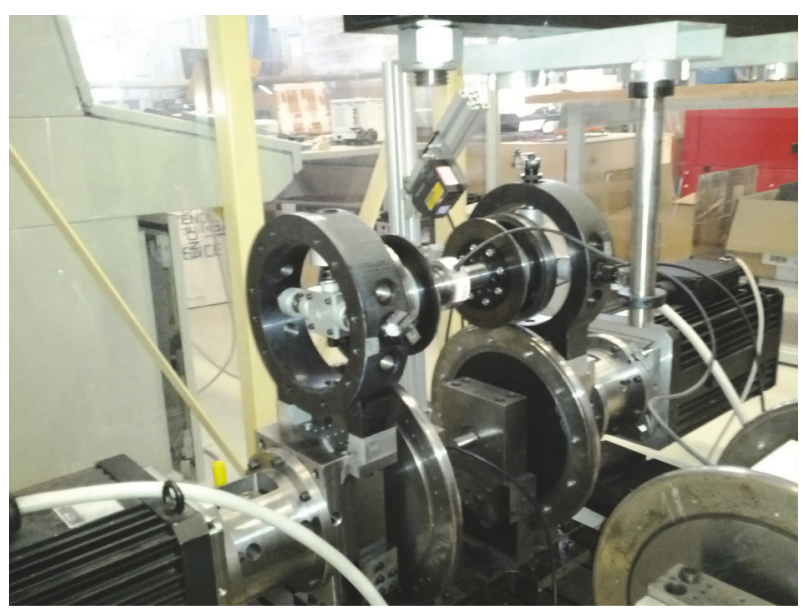

FIGURE 1: Roller-rig with a single suspended wheelset.

included in one single dynamic link library (.dll), which is called by the Simpack executables.

2.3. Experimental Configuration. Since the evaluation of the contact forces is very complex, it is necessary to perform the tests under well-known environmental conditions and this requirement can be easily achieved by using a real scale or a reduced scale roller-rig. The former allows a direct comparison with the real vehicle, while the latter requires a similitude model to convert the measured quantities into equivalent real scale values [18]. The work shows in Section 3.3 the use of a scaled roller-rig to measure the friction forces in different working conditions.

The roller-rig used in this work [19] is composed of a single wheelset supported by two independent rotating rollers. The wheelset is connected to the bench structure by means of a single stage suspension. The rollers are driven by two motors, which can be controlled independently. The suspension is composed of two vertical helical springs and four longitudinal springs.

The experimental device, shown in Figure 1, allows measuring the friction forces with a good precision and evaluating the adhesion conditions. In fact, the device allows measuring the friction coefficient as a function of the roller velocity and slip. The contact conditions between the wheel and the rail can be modified by injecting water or oil into the contact area.

The experimental configuration of the test rig is shown schematically in Figure 2, where the two rollers, which represent the rails, are independent and connected to two different motors, which can be driven with independent controls. The torque exerted by the two motors pass through the wheelset generating opposite friction forces on the two wheels, as shown in [20].

In this way, one wheel works in traction condition, while the other works in braking condition, and this allows studying and comparing the two phenomena simultaneously.

The test rig is equipped with different sensors: two torquemeters (maximum torque $200 \mathrm{Nm}$, nonlinearity $<0.3 \%$ of the rated output) arranged between each motor and the corresponding roller, used to measure the torque applied by the motor; there are three high resolution encoders (Heidenhain ECN 1325, with a resolution of 25 bit, an accuracy of \pm 20 arcseconds, and a clock frequency of $16 \mathrm{MHz}$ ), one for each motor and one on the wheelset. The encoder on the motor can be used for accurate angular velocity measurements or to control the motors in closed loop with respect to the angular velocity or the angular position.

The loads applied to the wheelset through the suspension elements are constantly measured using load cells (capacity of $1000 \mathrm{lbs}$, nonlinearity $<0.125 \%$ of the rated output) arranged between the suspensions and the fixed frame. Finally the test rig is equipped with high resolution laser sensors (Keyence LK-G82, with a repeatability of $0.2 \mathrm{um}$ and a sampling frequency range of $1-50 \mathrm{kHz}$ ) used to measure the wheelset position in terms of lateral and longitudinal displacements and to assess the yaw angle of the wheelset.

This experimental configuration allows simulating the behaviour of a vehicle running on a straight track during traction and braking, where the longitudinal creepage prevails, and is suitable to perform test on braking and adhesion. To prevent relevant transversal translation or yaw rotation of the wheelset, high stiffness values are used for the longitudinal and lateral direction.

Two control methods are possible for the motors: closed loop velocity control and torque control.

2.3.1. Closed Loop Velocity Control. This method operates by imposing different velocities on the two rollers and generating the desired creepage. The control starts with the same angular velocity on the two rollers and then the velocity of one of the motors is reduced.

The test rig allows switching from a condition of pure rolling, where the speeds of the right and the left rollers are the same, to a condition of controlled creepage, where the speeds are different.

The last condition is obtained imposing a steady angular velocity on the left roller motor, varying continuously the velocity of the right one, and decreasing its value until the desired maximum creepage value is achieved. The creepage is therefore controlled and the experimental measurement configuration allows measuring the longitudinal force for each creepage level.

The longitudinal creepages for the right and the left wheel can be calculated using (13) and (14), where $\omega_{\mathrm{Rr}}$ and $\omega_{\mathrm{Lr}}$ are the angular velocities of the right and left rollers, while $\omega_{\mathrm{w}}$ is the angular velocity of the wheelset.

$$
\begin{gathered}
\xi_{\mathrm{R}}=\frac{\omega_{\mathrm{Rr}} R_{R r}-\omega_{w} R_{R w}}{\omega_{\mathrm{Rr}} R_{R r}} \\
\xi_{\mathrm{L}}=\frac{\omega_{\mathrm{Lr}} R_{L r}-\omega_{w} R_{\mathrm{L} w}}{\omega_{\mathrm{Lr}} R_{\mathrm{Lr}}}
\end{gathered}
$$

In (13) and (14) the subscript $\mathrm{R}$ indicates the right side while the subscript $L$ indicates the left side. Instead, the subscripts $\mathrm{w}$ and $\mathrm{r}$ are used to denote the wheelset and the rollers, respectively. During the experimental test the wheelset is kept 


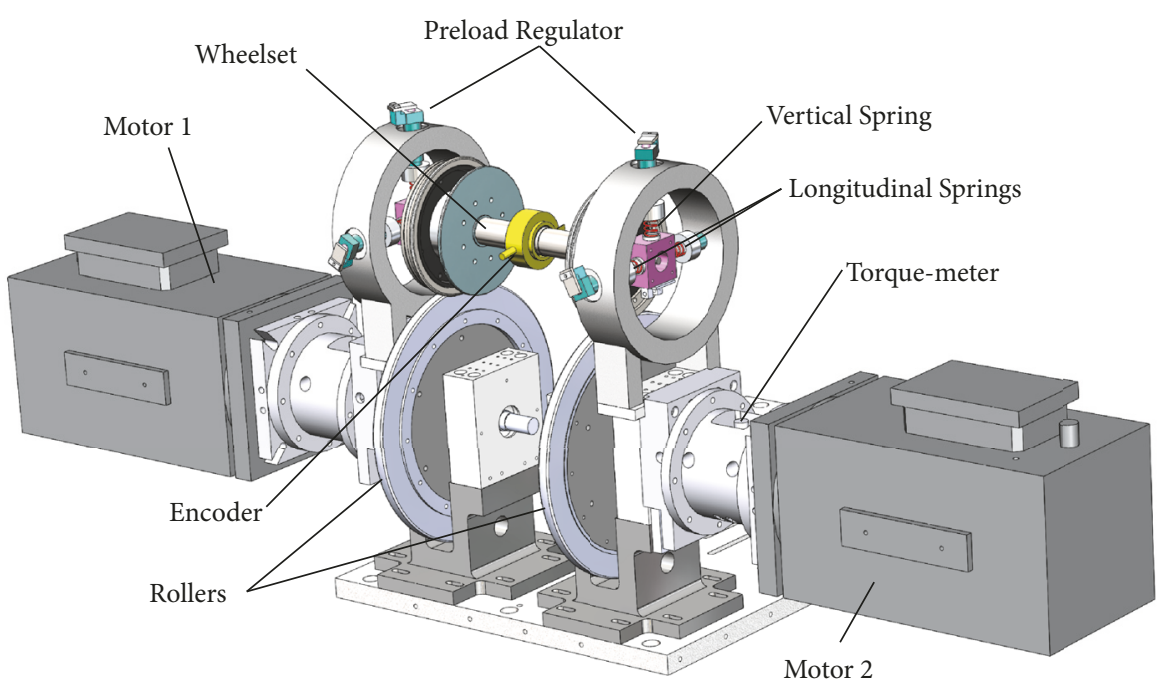

FIgURE 2: Motors and sensors disposition on the roller-rig.

centred with respect to the rollers; therefore, the wheelset rolling radii $R_{R w}$ and $R_{L w}$ can be considered equal, and the same consideration is also true for the right and the left rolling radii of the rollers $R_{R r}$ and $R_{L r}$. According to this hypothesis, (16) and (17) can be used to determine the longitudinal creepages.

$$
\begin{aligned}
& \xi_{\mathrm{R}}=\frac{\omega_{\mathrm{Rr}}-\omega_{\mathrm{Lr}}}{2 \omega_{\mathrm{Rr}}} \\
& \xi_{\mathrm{L}}=\frac{\omega_{\mathrm{Lr}}-\omega_{\mathrm{Rr}}}{2 \omega_{\mathrm{Lr}}}
\end{aligned}
$$

Moreover, since during the tests on roller-rig the wheelset is centred, the lateral creepage vanishes $\left(\eta_{\mathrm{R}, \mathrm{L}}=0\right)$, while a constant value of the spin creepage must be considered, due to the wheel conicity.

In this case (wheelset on roller-rig) the spin creepage can be calculated according to

$$
\phi_{\mathrm{R}, \mathrm{L}}= \pm \frac{\gamma_{\mathrm{R}}+\gamma_{\mathrm{L}}}{2} \cdot\left(\frac{1}{\mathrm{R}_{\mathrm{Rw}, \mathrm{Lw}}}+\frac{1}{\mathrm{R}_{\mathrm{Rr}, \mathrm{Lr}}}\right)
$$

where $\gamma_{\mathrm{L}}, \gamma_{\mathrm{R}}$ are the right and left contact angles depending on the profiles of wheel and roller $\left(\gamma_{\mathrm{L}}=\gamma_{\mathrm{R}}\right.$ when the wheelset is centred). In case of the test carried out on in this paper, the typical European profiles are used for wheel and rail, in scale 1:4 (UIC60 canted 1/20 for the rail and S1002 for the wheel). The roller profile transversal curvature is modified to compensate the inherent error arising from the use of a roller-rig and to preserve the aspect ratio of the contact area versus those obtained on a real track $[19,21]$. Table 3 shows the relevant dimensions adopted during the tests on the rollerrig. Different axle loads $\left(N_{1}, N_{2}, N_{3}\right)$ are simulated by acting on the preload systems of the test rig. Table 3 shows the calculated dimension of the contact area for the profiles used during the experimental tests.

All the quantities in the prototype used on the roller-rig are scaled according to the similitude approach proposed by Jashinski $[18,19]$.
A set of scaling factors allows converting the values of the quantities measured during the test on the scaled prototype into the corresponding values estimated for the real vehicle. A scale factor of 1:4 $\left(\varphi_{l}=4\right)$ is considered for the length. The forces are subsequently scaled by a factor $\varphi_{f}=\varphi_{l}^{3}=64$.

This means that the tests correspond to a real vehicle with an axle load of about 6 tonnes. The used approach maintains a unit scale factor for acceleration, while velocity and time are scaled by a factor $\varphi_{t}=\varphi_{v}=\varphi_{l}^{0.5}=2$. The angular velocity is scaled by a factor $\varphi_{\omega}=\varphi_{l}^{-0.5}=0.5$.

2.3.2. Torque Control. The alternative method consists in keeping the velocity of one of the motors constant and applying a resistant torque on the second motor. The resistant torque is gradually increased up to the sliding condition.

The torque control method is more reliable for low creepage values, while it becomes unstable when the adhesion limit is reached. This method can be conveniently used to define the behaviour of the creep-traction force curve close to the origin, where the adhesion law is quite linear.

The measurement of the signals and calculation of the quantities of interest for the test can be performed according to the same procedures described for the case of the closed loop velocity control.

Both control methods were used during the tests and compared with the numerical results.

\section{Results and Discussion}

The performances of the proposed method are estimated considering three different comparisons, which are shown in this section. At first, in Section 3.1, the results of the algorithm are directly compared with those obtained from the Polach's method and from FASTSIM, using a Matlab routine where all the algorithms are implemented. In Section 3.2, the three methods are compared using the Simpack multibody code (FASTSIM and Polach are already implemented in Simpack 
TABLE 3: Geometrical data of the roller-rig used during the tests.

\begin{tabular}{|c|c|c|c|}
\hline Symbol & Definition & \multicolumn{2}{|c|}{ Value } \\
\hline $\mathrm{R}_{\mathrm{r}}$ & Nominal roller radius & \multicolumn{2}{|c|}{$185 \mathrm{~mm}$} \\
\hline $\mathrm{R}_{\mathrm{w}}$ & Nominal wheel radius (scaled 1:4) & \multicolumn{2}{|c|}{$95 \mathrm{~mm}$} \\
\hline $\mathrm{N}$ & Axle-Load (N1, N2) & $848 \mathrm{~N}$ & $946 \mathrm{~N}$ \\
\hline $\mathrm{a}$ & Contact ellipse longitudinal semi-axis & $0.588 \mathrm{~mm}$ & $0.610 \mathrm{~mm}$ \\
\hline $\mathrm{b}$ & Contact ellipse lateral semi-axis & $0.525 \mathrm{~mm}$ & $0.544 \mathrm{~mm}$ \\
\hline
\end{tabular}
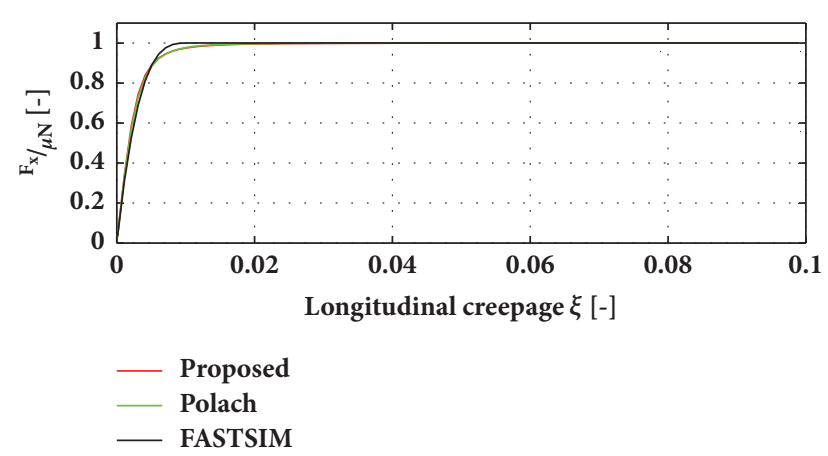

FIGURE 3: Longitudinal force versus pure longitudinal creepage.

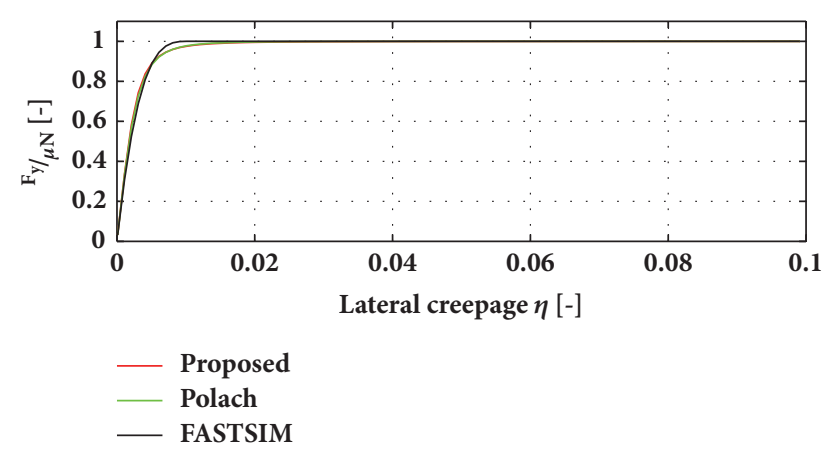

FIGURE 4: Lateral force versus pure lateral creepage.

2014 used for the test) considering a model of a real vehicle running on track. Finally, in Section 3.3, the results of the code are compared to experimental tests performed on rollerrig using a scaled prototype.

3.1. Numerical Results. The proposed algorithm was implemented in a Matlab routine and compared with FASTSIM and with the Polach's method. In order to make the comparison in the same condition, the same geometrical and material properties were assumed for the three methods. A material with a Poisson's ratio $\nu=0.25$ and a shear modulus $G=$ $8.4 \cdot 10^{9} \mathrm{Nm}^{-2}$ was adopted for all the tests, in agreement with [7]. A normal force of $100 \mathrm{kN}$ and a constant friction coefficient $\mu=0.35$ were used. In order to analyze the behaviour of the friction forces as a function of the creepage, at first, the semiaxes of the contact ellipse were assumed as constant, with $a=b=6 \mathrm{~mm}$. The Kalker's coefficients $C_{i j}$ for this case are shown in the first line of Table 3. Figures 3 and

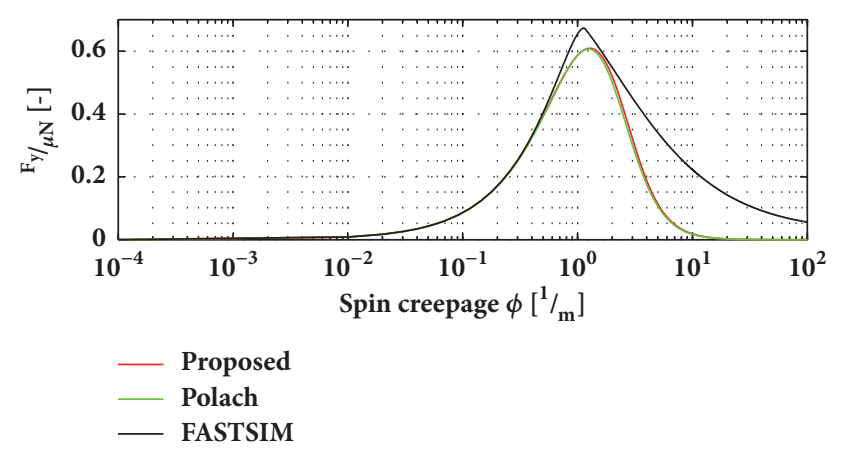

FIGURE 5: Lateral force versus pure spin creepage.

11 show the behaviour of the lateral and longitudinal forces versus the kinematical creepages for the three methods.

Figure 3 shows the behaviour of the longitudinal tangential force as a function of the longitudinal creepage in case of absence of other creepages. The results of the proposed method are shown in red colour, the results of FASTSIM in black, and the results of the Polach's method in green; the same colour convention is adopted for all the figures.

Figure 4 shows the behaviour of the lateral force versus the lateral creepage in absence of other creepages, and Figure 5 shows the behaviour of the lateral force versus the spin creepage in absence of other creepages. Figure 5 is plotted using a logarithmic scale in the $\mathrm{x}$ axis (spin creepage) due to the wide range along this axis. The comparison between the proposed method and the Polach's method is in very good agreement, since the parameters were tuned over the Polach's method. The FASTSIM algorithm was simulated using a discretization of the contact area of 50x50 elements in order to achieve a good accuracy (usually 20x20 is used in MBS codes).

In order to investigate the behaviour in a more realistic case, where more creepages are simultaneously present, Figures 6 and 7 show the longitudinal force versus the longitudinal creepage, in presence of a lateral creepage $(\eta=$ $0.003)$ and spin creepage $(\phi=0.2)$, respectively. When both longitudinal and lateral creepage exist, the proposed method is more similar to FASTSIM, while when longitudinal and spin creepage are both present, the proposed method is more similar to the Polach's method.

Figures 8 and 9 analyze the behaviour of the lateral force versus the lateral creepage in presence of longitudinal $(\xi=$ $0.003)$ and spin creepage $(\phi=0.1)$, respectively. In case of lateral and longitudinal creepage the three methods are quite 


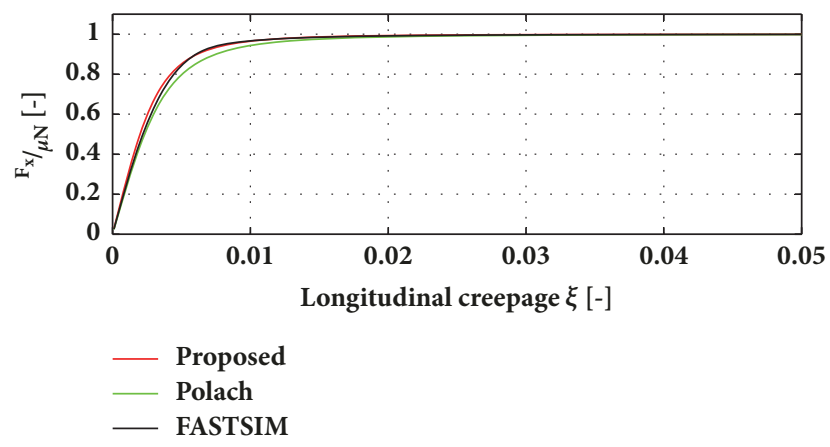

FIGURE 6: Longitudinal force versus longitudinal creepage in presence of a constant lateral creepage $(\eta=0.003)$.

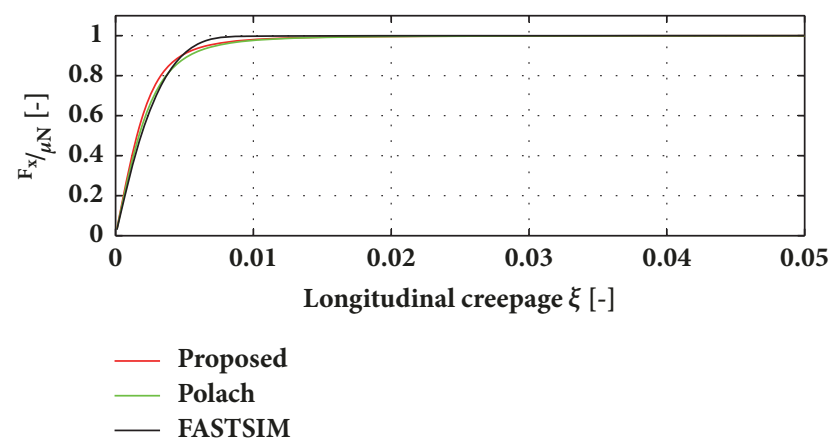

FIGURE 7: Longitudinal force versus longitudinal creepage in presence of a constant spin creepage $(\phi=0.2)$.

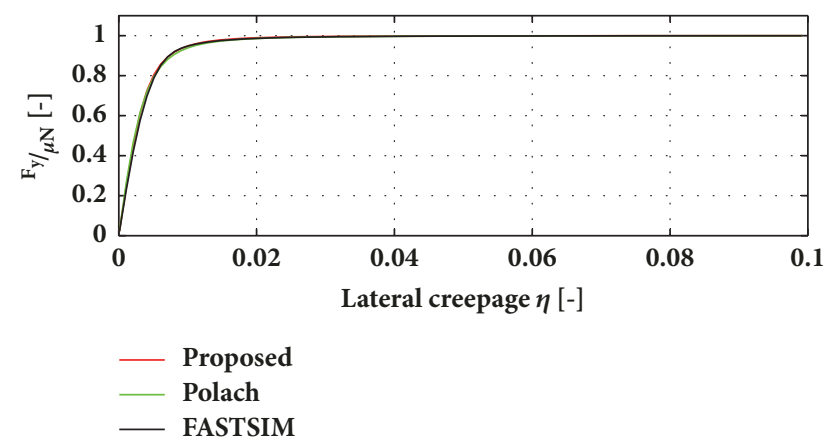

FigURE 8: Lateral force versus lateral creepage in presence of a constant longitudinal creepage $(\xi=0.003)$.

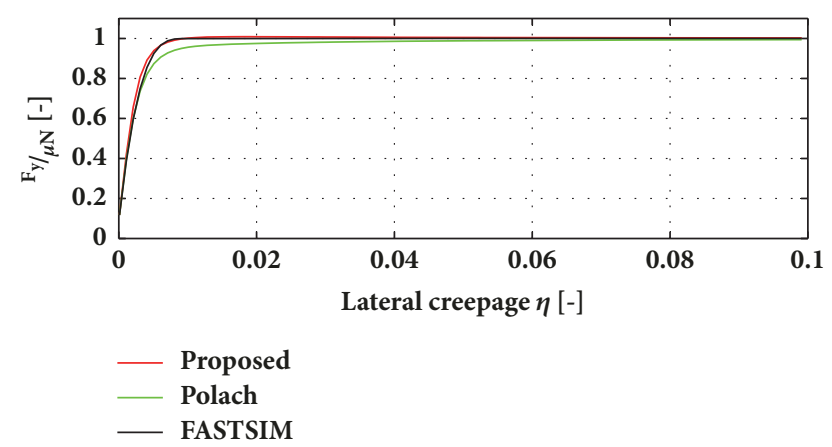

FIGURE 9: Lateral force versus lateral creepage in presence of a constant spin creepage $(\phi=0.1)$.

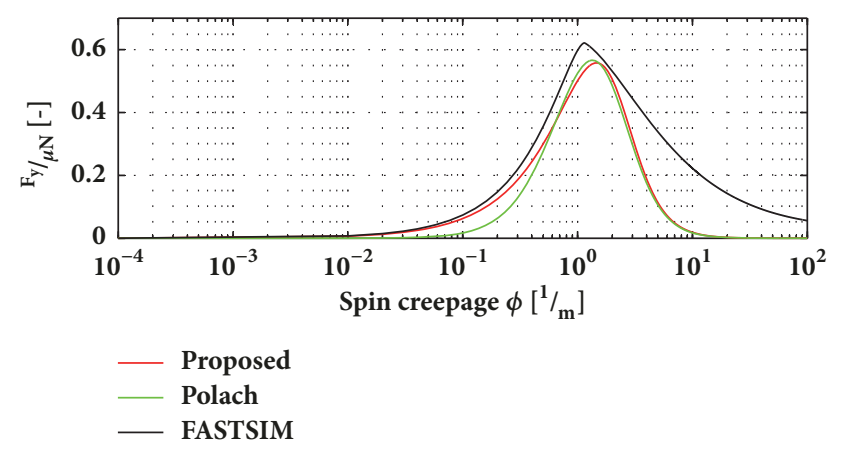

FIGURE 10: Lateral force versus spin creepage in presence of a constant longitudinal creepage $(\xi=0.003)$.

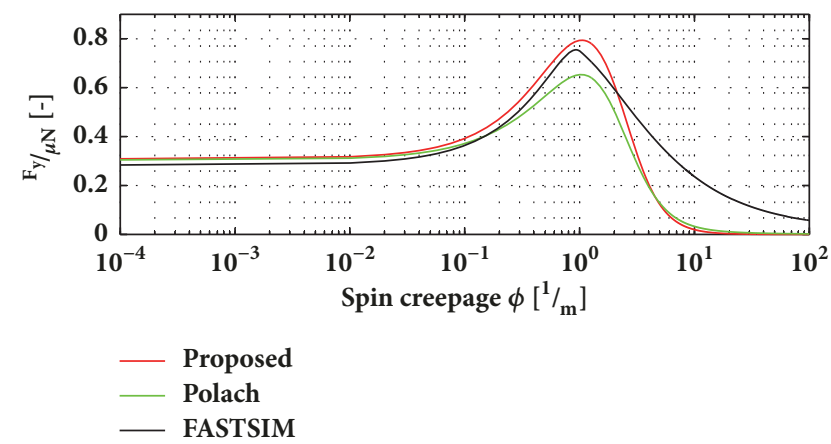

FIGURE 11: Lateral force versus spin creepage in presence of a constant lateral creepage $(\eta=0.001)$.

similar, while in case of presence of lateral and spin creepage, the proposed method is in better agreement with FASTSIM.

Figures 10 and 11 analyze the behaviour of the lateral force versus the spin creepage in presence of longitudinal $(\xi=$ $0.003)$ and lateral creepage $(\phi=0.001)$, respectively. In this case the proposed method appears in better agreement with the Polach's method.

Since the behaviour shown in the previous tables is calculated with a single value of the $\mathrm{a} / \mathrm{b}$ ratio, also the effect of the shape of the contact area was investigated for the three methods, by reproducing the test cases proposed by Polach in [7] as described in Table 4.

The results of the test cases for the three methods for $\mu=0.3$ are shown in Table 5, where it is evident that the three methods are in good agreement.

The numerical efficiency of the methods was compared and the results are shown in Table 6. In order to obtain the results, all the algorithms are written as Matlab routines. The time indicated in Table 6 is the one required to generate the data used for the plots shown in Figures 3 and 11.

The results are shown in percentage, where $100 \%$ of time is attributed to FASTSIM (discretized 50x50). The simulations of this section were performed on an Intel core i5-2400@3.10 GHz processor with 16 GB of DDR3 RAM, running Windows 7 Pro 64 bit (4 Cores).

3.2. Comparison with a Full Vehicle Simulation. Multibody codes are usually adopted to simulate the dynamic of railway 
TABLE 4: Data adopted for the comparison of the methods, from [1].

\begin{tabular}{lcccccccc}
\hline Case & $\mathrm{a}$ & $\mathrm{b}$ & $\mathrm{C}_{11}$ & $\mathrm{C}_{22}$ & $\mathrm{C}_{23}$ & $\xi$ & \multicolumn{1}{c}{$\eta$} \\
\hline 1 & $6 \times 10^{-3} \mathrm{~m}$ & $6 \times 10^{-3} \mathrm{~m}$ & 4.12 & 3.67 & 1.47 & 0.004 & 0 & $0.0 \mathrm{~m}^{-1}$ \\
2 & $6 \times 10^{-3} \mathrm{~m}$ & $6 \times 10^{-3} \mathrm{~m}$ & 4.12 & 3.67 & 1.47 & 0 & 0.004 & 0 \\
3 & $6 \times 10^{-3} \mathrm{~m}$ & $6 \times 10^{-3} \mathrm{~m}$ & 4.12 & 3.67 & 1.47 & 0 & $0.004 \mathrm{~m}^{-1}$ \\
4 & $6 \times 10^{-3} \mathrm{~m}$ & $6 \times 10^{-3} \mathrm{~m}$ & 4.12 & 3.67 & 1.47 & 0.002 & 0.002 & $0.002 \mathrm{~m}^{-1}$ \\
5 & $6 \times 10^{-3} \mathrm{~m}$ & $6 \times 10^{-3} \mathrm{~m}$ & 4.12 & 3.67 & 1.47 & 0.004 & 0.006 & $0 \mathrm{~m}^{-1}$ \\
6 & $6 \times 10^{-3} \mathrm{~m}$ & $6 \times 10^{-3} \mathrm{~m}$ & 4.12 & 3.67 & 1.47 & 0.00005 & 0.004 & $0.008 \mathrm{~m}^{-1}$ \\
7 & $6 \times 10^{-3} \mathrm{~m}$ & $6 \times 10^{-3} \mathrm{~m}$ & 4.12 & 3.67 & 1.47 & -0.00005 & 0.004 & $0.008 \mathrm{~m}^{-1}$ \\
8 & $6 \times 10^{-3} \mathrm{~m}$ & $6 \times 10^{-3} \mathrm{~m}$ & 4.12 & 3.67 & 1.47 & -0.00005 & -0.004 & $0.8 \mathrm{~m}^{-1}$ \\
9 & $6 \times 10^{-3} \mathrm{~m}$ & $6 \times 10^{-3} \mathrm{~m}$ & 4.12 & 3.67 & 1.47 & 0.00005 & -0.004 & $0.008 \mathrm{~m}^{-1}$ \\
10 & $7.5 \times 10^{-3} \mathrm{~m}$ & $1.5 \times 10^{-3} \mathrm{~m}$ & 7.78 & 8.14 & 6.63 & 0.002 & 0.002 & 0 \\
11 & $7.5 \times 10^{-3} \mathrm{~m}$ & $1.5 \times 10^{-3} \mathrm{~m}$ & 7.78 & 8.14 & 6.63 & 0 & 0.002 & $0.002 \mathrm{~m}^{-1}$ \\
12 & $7.5 \times 10^{-3} \mathrm{~m}$ & $1.5 \times 10^{-3} \mathrm{~m}$ & 7.78 & 8.14 & 6.63 & 0.002 & 0 & $0.002 \mathrm{~m}^{-1}$ \\
13 & $1.5 \times 10^{-3} \mathrm{~m}$ & $7.5 \times 10^{-3} \mathrm{~m}$ & 3.37 & 2.63 & 0.603 & 0.002 & 0.002 & $0 \mathrm{~m}^{-1}$ \\
14 & $1.5 \times 10^{-3} \mathrm{~m}$ & $7.5 \times 10^{-3} \mathrm{~m}$ & 3.37 & 2.63 & 0.603 & 0 & 0.002 & $0.002 \mathrm{~m}^{-1}$ \\
15 & $1.5 \times 10^{-3} \mathrm{~m}$ & $7.5 \times 10^{-3} \mathrm{~m}$ & 3.37 & 2.63 & 0.603 & 0.002 & 0 & $0.002 \mathrm{~m}^{-1}$ \\
\hline
\end{tabular}

TABLE 5: Results of the comparison in terms of tangential forces.

\begin{tabular}{|c|c|c|c|c|c|c|}
\hline \multirow{2}{*}{ Case } & \multicolumn{2}{|c|}{ Polach } & \multicolumn{2}{|c|}{ FASTSIM } & \multicolumn{2}{|c|}{ Proposed } \\
\hline & $\mathrm{Fx}$ & Fy & Fx & Fy & Fx & Fy \\
\hline 1 & $-26732 \mathrm{~N}$ & $0 \mathrm{~N}$ & $-27008 \mathrm{~N}$ & $0 \mathrm{~N}$ & $-2.6930 \mathrm{~N}$ & 0 \\
\hline 2 & $0 \mathrm{~N}$ & $-25872 \mathrm{~N}$ & $0 \mathrm{~N}$ & $-25780 \mathrm{~N}$ & $0 \mathrm{~N}$ & $-26150 \mathrm{~N}$ \\
\hline 3 & $0 \mathrm{~N}$ & $-107 \mathrm{~N}$ & $0 \mathrm{~N}$ & $-107 \mathrm{~N}$ & $0 \mathrm{~N}$ & $-107 \mathrm{~N}$ \\
\hline 4 & $-16362 \mathrm{~N}$ & $-16398 \mathrm{~N}$ & $-16630 \mathrm{~N}$ & $-14859 \mathrm{~N}$ & $-18199 \mathrm{~N}$ & $-16250 \mathrm{~N}$ \\
\hline 5 & $-16098 \mathrm{~N}$ & $-24147 \mathrm{~N}$ & $-17928 \mathrm{~N}$ & $-23955 \mathrm{~N}$ & $-18241 \mathrm{~N}$ & $-24373 \mathrm{~N}$ \\
\hline 6 & $-321 \mathrm{~N}$ & $-25834 \mathrm{~N}$ & $-361 \mathrm{~N}$ & $-25887 \mathrm{~N}$ & $-367 N$ & $-26276 \mathrm{~N}$ \\
\hline 7 & $321 \mathrm{~N}$ & $-25834 \mathrm{~N}$ & $361 \mathrm{~N}$ & $-25887 \mathrm{~N}$ & $367 \mathrm{~N}$ & $-26276 \mathrm{~N}$ \\
\hline 8 & $323 \mathrm{~N}$ & $8259 \mathrm{~N}$ & $285 \mathrm{~N}$ & $9364 \mathrm{~N}$ & $335 \mathrm{~N}$ & $-12414 \mathrm{~N}$ \\
\hline 9 & $-321 \mathrm{~N}$ & $25834 \mathrm{~N}$ & $362 \mathrm{~N}$ & $25668 \mathrm{~N}$ & $367 \mathrm{~N}$ & $26024 \mathrm{~N}$ \\
\hline 10 & $-12606 \mathrm{~N}$ & $-12606 \mathrm{~N}$ & $-11409 \mathrm{~N}$ & $-11937 \mathrm{~N}$ & $-12850 \mathrm{~N}$ & $-13445 \mathrm{~N}$ \\
\hline 11 & $0 \mathrm{~N}$ & $-13954 \mathrm{~N}$ & $0 \mathrm{~N}$ & $-12866 \mathrm{~N}$ & $0 \mathrm{~N}$ & $-14292 \mathrm{~N}$ \\
\hline 12 & $-13421 \mathrm{~N}$ & $-0.3 \mathrm{~N}$ & $-12361 \mathrm{~N}$ & $-40 \mathrm{~N}$ & $-1.3721 \mathrm{~N}$ & $-39 \mathrm{~N}$ \\
\hline 13 & $-5549 \mathrm{~N}$ & $-5549 \mathrm{~N}$ & $-5796 \mathrm{~N}$ & $-4523 \mathrm{~N}$ & $-6272 \mathrm{~N}$ & $-4895 \mathrm{~N}$ \\
\hline 14 & $0 \mathrm{~N}$ & $-4919 \mathrm{~N}$ & $0 \mathrm{~N}$ & $-4.701 \mathrm{~N}$ & $0 \mathrm{~N}$ & $-4947 \mathrm{~N}$ \\
\hline 15 & $-6254 \mathrm{~N}$ & $0 \mathrm{~N}$ & $-5916 \mathrm{~N}$ & $-3.8 \mathrm{~N}$ & $-6306 \mathrm{~N}$ & $-3.8 \mathrm{~N}$ \\
\hline
\end{tabular}

TABLE 6: Comparison of the computational times.

\begin{tabular}{lccc}
\hline Method & Proposed & Polach & FASTSIM \\
\hline Time [\%] & 5.5 & 7.2 & 100 \\
\hline
\end{tabular}

vehicles. Therefore, this paragraph compares the results of the three methods using a numerical model of the complete vehicle, developed using the Simpack multibody code. The longitudinal and lateral friction forces at the wheel/rail contact are considered for the comparison.

As previously described, the algorithm for calculating friction forces described in this paper has been included in the Simpack library. The simulations consider a $2.5 \mathrm{~km}$ long section of the Aosta-Pré-Saint-Didier track, where the considered vehicle usually runs.
Figures 12 and 13, respectively, show the longitudinal and lateral friction force, measured on the right wheel of the leading wheelset. This track section includes five curves, whose curvature radii are shown in the plot. Regarding the longitudinal friction force, we can remark that the three algorithms are in general in good agreement, while some small differences can be observed considering the two curves with curve radius of $-1300 \mathrm{~m}$ and $250 \mathrm{~m}$. In the first case, the proposed algorithm agrees with the one proposed by Polach, while in the second case it fits the FASTSIM method. More important differences can be observed considering the lateral friction force, and in this case the proposed algorithm mostly agrees with FASTSIM, with the exception of the third and the last curve, where it is more similar to the Polach's method.

In conclusion, since the results of the proposed method fall mainly between the other methods, considering that the 


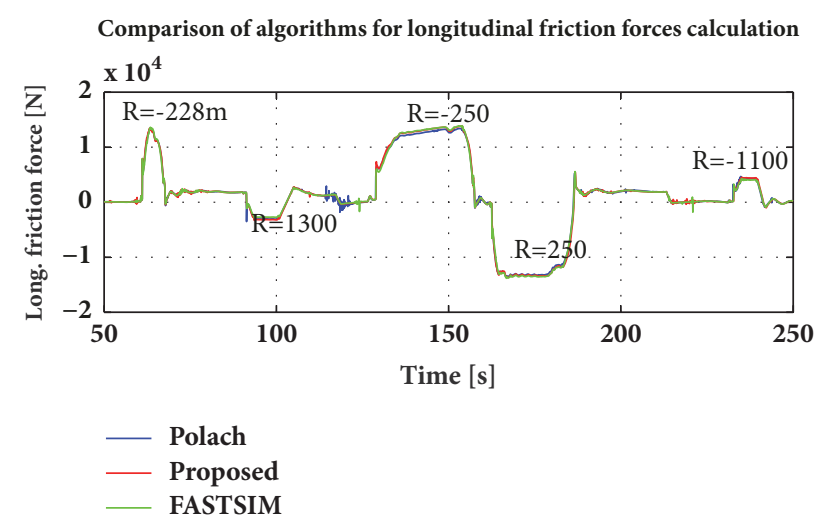

FIGURE 12: Longitudinal friction forces obtained on a section of the Aosta-Pré-Saint-Didier track.

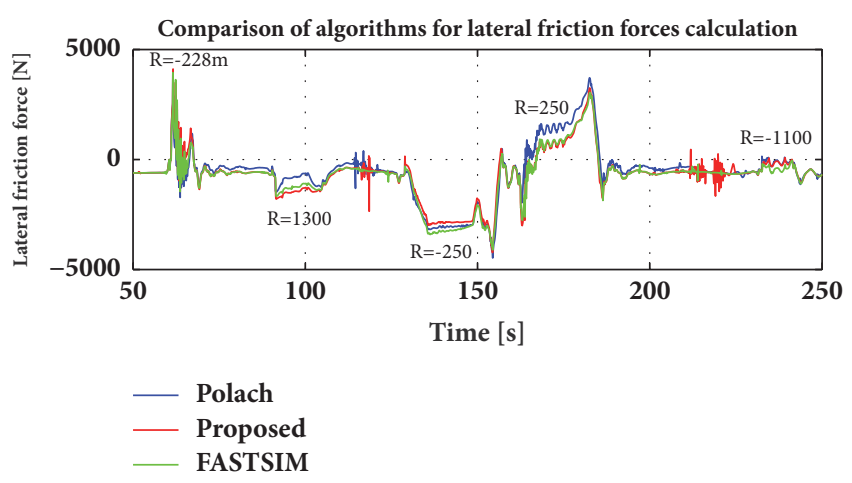

FIGURE 13: Lateral friction forces obtained on a section of the Aosta-Pré-Saint-Didier track.

TABLE 7: Comparison of calculation time and number of rejected steps considering a simulation of $250 \mathrm{~s}$.

\begin{tabular}{lcc}
\hline Method & Number of rejected steps & Wall clock time [s] \\
\hline FASTSIM & 888 & 102.38 \\
Polach & 1047 & 69.45 \\
Proposed & 630 & 60.08 \\
\hline
\end{tabular}

differences in the worst case are quite small, we can state that the proposed method is substantially equivalent to the standard methods in a multibody simulation.

Table 7 compares the performances of the three algorithms in terms of computational time. The results consider a simulation of $250 \mathrm{~s}$. The proposed algorithm is $15 \%$ faster than Polach and $70 \%$ faster than FASTSIM, allowing a better convergence and reducing the number of rejected time steps. The simulations were performed on an Intel Xeon E5-2630L@1.8 GHz with 128 GB of RAM, running Windows Server 200864 bit (32 Cores).

3.3. Experimental Results. This last section shows the experimental validation of the proposed method, which was carried out using a scaled prototype on roller-rig. The dynamic of a vehicle on a real track is quantitatively different from, but qualitatively similar to, that on a scaled roller-rig. This means that the same phenomenon can be observed, but corrections are needed (scaling factors, different creepages) to compare the results on the real track to those obtained on a rollerrig. Furthermore the roller does not allow reproducing the behaviour of the vehicle on curves. For this reason, in the previous sections of the paper the methods are compared considering numerical simulation that can be applied to a real track. The tests performed on the roller-rig are instead important to reproduce the behaviour of the friction forces in nonsteady condition.

Since the tribological behaviour on roller-rig is the same as in reality, it is possible to adopt these tests to determine the shape of the adhesion curve and estimate the coefficients to be used in the proposed method, so as to faithfully copy the experimental trend. Remember that in the first part of the work the coefficients of the method have been set in order to reproduce the same behaviour of the Polach's method and FASTSIM. In this section we intend to illustrate how it is possible to adapt the method to experimental results, which in this case are obtained on a roller-rig, but which could possibly be obtained by on-track tests.

The test was performed on the roller-rig, with a single suspended wheelset, considering an angular velocity of $358 \mathrm{rpm}$ and a vertical load of 48 and $96 \mathrm{Kg}$. Since the tests were performed on a 1:4 scaled system, this corresponds in real scale to a vehicle running at $50 \mathrm{~km} / \mathrm{h}$ with an axle load of $3072 / 6144 \mathrm{Kg}$. The tests were performed in dry condition. During the test, the tangential force is calculated from the measured value of the torque applied to the rollers, by dividing the torque value by the roller radius. The actual friction coefficient can be calculated by dividing the value of the tangential force by the normal load acting on the wheel.

Figure 14 shows the experimental results obtained controlling the roller-rig in torque and velocity mode compared with the numerical simulation of the same case using the proposed method.

The tests performed in torque and velocity control provide the same behaviour of the curves close to the origin, where the linear law applies. At higher creepage the behaviours differ in terms of adhesion coefficient ( $\mathrm{f}=0.42$ in case of torque control and 0.45 in case of velocity control) and both curves exhibit a reduction of adhesion with increasing creepage.

Figure 14 also shows three different numerical curves, all obtained with the proposed algorithm with different parameters. The curves are normalized with respect to the adhesion limit. The third numerical curve (in the legend) is obtained with the same parameters used to fit the Polach/FASTSIM algorithms in the previous section $\left(n=n_{1}=2.4\right)$. This curve shows the same initial slope of the experiments, but a different nonlinear behaviour. The second curve was introduced to better fit the nonlinear behaviour of the experimental data, and it is obtained by modifying only the main parameter $n=n_{1}=1.5$. This second curve is still unable to fit the experimental results for large creepage, as it does not allow reproducing the decreasing adhesion versus the creepage ("falling friction"). A good agreement could be obtained from the second curve by using a friction coefficient, that is variable with the creepage. Since the implementation of 


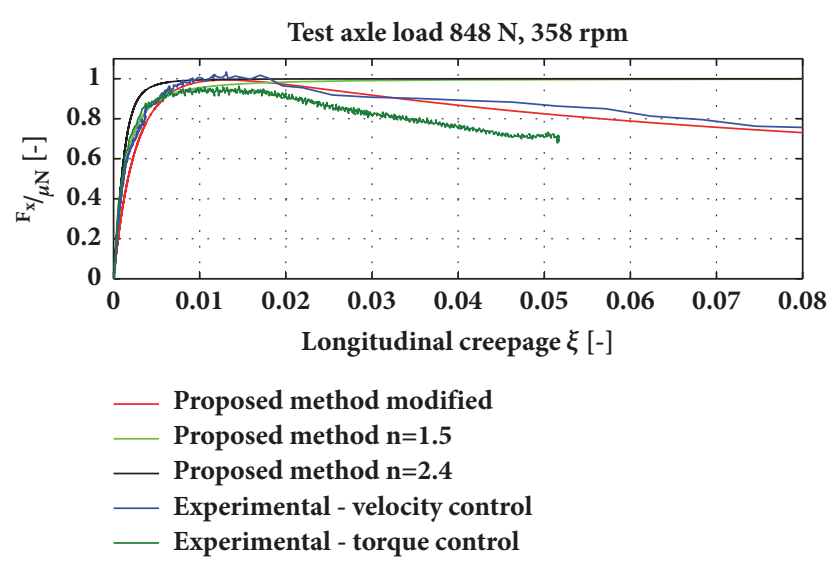

FIGURE 14: Normalized longitudinal tangential force on roller-rig, with an axle load of $848 \mathrm{~N}$ and a reference velocity of $358 \mathrm{rpm}$.

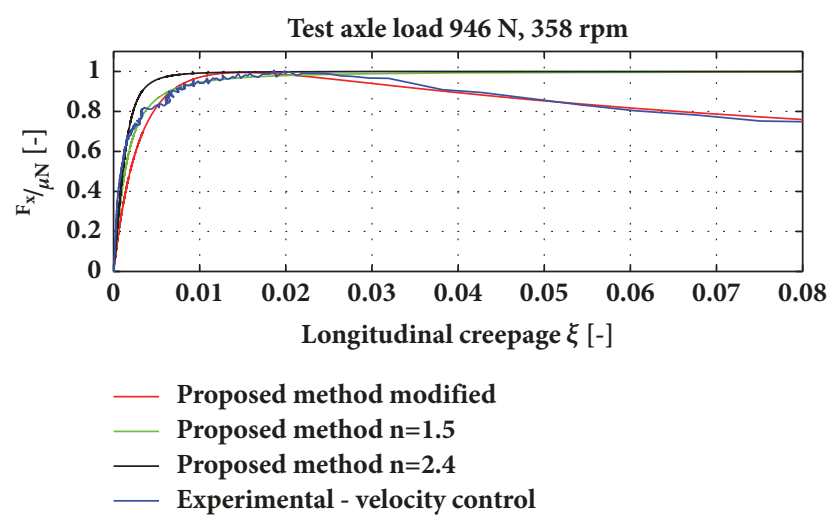

FIGURE 15: Normalized longitudinal tangential force on roller-rig, with an axle load of $946 \mathrm{~N}$ and a reference velocity of $358 \mathrm{rpm}$.

a variable friction coefficient implies additional functions and computational time, the first numerical curve (proposed method modified) is obtained only acting on the coefficients of the proposed method, which were modified in order to achieve a better compromise. The modified method adopts the following coefficients: $n=0.8, n_{1}=1.07$, and $n_{6}=5.2$, while the other coefficients are unchanged.

Figure 15 shows the comparison with a different axle load $(946 \mathrm{~N})$; the experimental test has been performed only in velocity control in this case. During this second test a different value of the friction coefficient was measured (0.49), showing an increment in adhesion with the axle load. Also in this case the modified algorithm fits the experimental curve with the same parameters used for the previous case.

\section{Conclusions}

The paper proposes a fast and simplified nonlinear method for calculating the tangential forces for wheel-rail and wheelroller contact. The algorithm allows, in fact, obtaining the longitudinal and lateral friction forces by means of two analytical equations. The work provides several comparisons of the proposed algorithm with respect to FASTSIM and the
Polach's method, which are commonly used in the literature for the evaluation of the wheel-rail friction forces. At first, the algorithms were compared in simple test cases, demonstrating a very good agreement, over a wide range of the variables of interest for the wheel/rail contact ( $\mathrm{a} / \mathrm{b}$ ratio, normal load). The algorithm was then included in a commercial multibody code and used to calculate the friction forces, considering a vehicle running on the track. In this case, it demonstrates a very good computational efficiency, by reducing the calculation time of the $15 \%$ with respect to Polach's method and of the $70 \%$ with respect to FASTSIM. The proposed algorithm was finally validated, by using experimental results obtained from a scaled prototype of a single suspended wheelset on a rollerrig. The algorithm, consisting of two analytical equations that are a direct function of the three kinematical creepages, allows easily adapting the characteristic of the adhesion curve by using a set of coefficients. For this capability, the algorithm proposed in this paper can be of particular interest to achieve a more realistic simulation of the behaviour of railway vehicle dynamic, especially when results of experimental tests are available.

\section{Data Availability}

The article does not use external data; all the methods or sources referenced in the article have been cited in the References.

\section{Conflicts of Interest}

The authors declare no conflicts of interest.

\section{Acknowledgments}

The authors declare that this work has been funded using only institutional funds of Politecnico di Torino (internal grants for base research).

\section{References}

[1] J. J. Kalker, "Wheel-rail rolling contact theory," Wear, vol. 144, no. 1-2, pp. 243-261, 1991.

[2] J. J. Kalker, Three-dimensional Elastic Bodies in Rolling Contact. Solid Mechanics and Its Applications, vol. 2, Kluwer Academic Publishers, Dordrecht, The Netherlands, 1990.

[3] J. P. Pascal and G. Sauvage, "The Available Methods to Calculate the Wheel/Rail Forces in Non Hertzian Contact Patches and Rail Damaging," Vehicle System Dynamics, vol. 22, no. 3-4, pp. 263-275, 1993.

[4] W. Schiehlen, "Benchmark problems from vehicle dynamics," Journal of Mechanical Science and Technology, vol. 29, no. 7, pp. 2601-2606, 2015.

[5] G. Schupp, "Simulation of Railway Vehicles: Necessities and Applications," Mechanics Based Design of Structures and Machines, vol. 31, no. 3, pp. 297-314, 2003.

[6] J. J. Kalker, "A Fast Algorithm for the Simplified Theory of Rolling Contact," Vehicle System Dynamics, vol. 11, no. 1, pp. 1-13, 1982. 
[7] O. Polach, "Fast wheel-rail forces calculation computer code," Vehicle System Dynamics, vol. 33, pp. 728-739, 1999.

[8] Z. Y. Shen, J. K. Hedrick, and J. A. Elkins, "A comparison of alternative creep force models for rail vehicle dynamic analysis," Vehicle System Dynamics, vol. 12, no. 1-3, pp. 79-78, 1983.

[9] H. Chollet, M. Sebes, and J. B. Ayasse, "Evolution from the HERtzian contact model to non-HERtzian conditions for fast dynamic simulations," in Solid Mechanics and its Applications, vol. 3, 2007.

[10] N. Bosso, A. Gugliotta, and N. Zampieri, "RTCONTACT: An efficient wheel-rail contact algorithm for real-time dynamic simulations," in Proceedings of the 2012 Joint Rail Conference, JRC 2012, pp. 195-204, Philadelphia, Penn, USA, April 2012.

[11] M. Sebès, L. Chevalier, J.-B. Ayasse, and H. Chollet, "A fastsimplified wheel-rail contact model consistent with perfect plastic materials," Vehicle System Dynamics, vol. 50, no. 9, pp. 1453-1471, 2012.

[12] E. A. H. Vollebregt, S. D. Iwnicki, G. Xie, and P. Shackleton, "Assessing the accuracy of different simplified frictional rolling contact algorithms," Vehicle System Dynamics, vol. 50, no. 1, pp. $1-7,2012$.

[13] O. Polach, "Creep forces in simulations of traction vehicles running on adhesion limit," Wear, vol. 258, no. 7-8, pp. 9921000, 2005.

[14] E. A. H. Vollebregt and P. Wilders, "FASTSIM2: A second-order accurate frictional rolling contact algorithm," Computational Mechanics, vol. 47, no. 1, pp. 105-116, 2011.

[15] E. A. H. Vollebregt, "Numerical modeling of measured railway creep versus creep-force curves with CONTACT," Wear, vol. 314, no. 1-2, pp. 87-95, 2014.

[16] E. A. H. Vollebregt, "FASTSIM with falling friction and friction memory," Notes on Numerical Fluid Mechanics and Multidisciplinary Design, vol. 126, pp. 425-432, 2014.

[17] M. Spiryagin, O. Polach, and C. Cole, "Creep force modelling for rail traction vehicles based on the Fastsim algorithm," Vehicle System Dynamics, vol. 51, no. 11, pp. 1765-1783, 2013.

[18] A. Jaschinski, H. Chollet, S. Iwnicki, A. Wickens, and J. V. Würzen, "The application of roller rigs to railway vehicle dynamics," Vehicle System Dynamics, vol. 31, no. 5-6, pp. 345392, 1999.

[19] N. Bosso, M. Spiryagin, A. Gugliotta, and A. Somà, "Mechatronic modeling of real-time wheel-rail contact," Mechatronic Modeling of Real-Time Wheel-Rail Contact, vol. 9783642362460 , pp. 1-119, 2013.

[20] N. Bosso, A. Gugliotta, and N. Zampieri, "Strategies to simulate wheel-rail adhesion in degraded conditions using a roller-rig," Vehicle System Dynamics, vol. 53, no. 5, pp. 619-634, 2015.

[21] N. Bosso, A. Gugliotta, and A. Somà, "Dynamic behavior of a railway wheelset on a roller rig versus tangent track," Shock and Vibration, vol. 11, no. 3-4, pp. 467-492, 2004. 


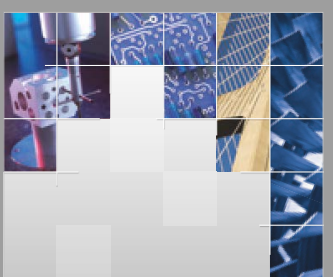

\section{Enfincering}
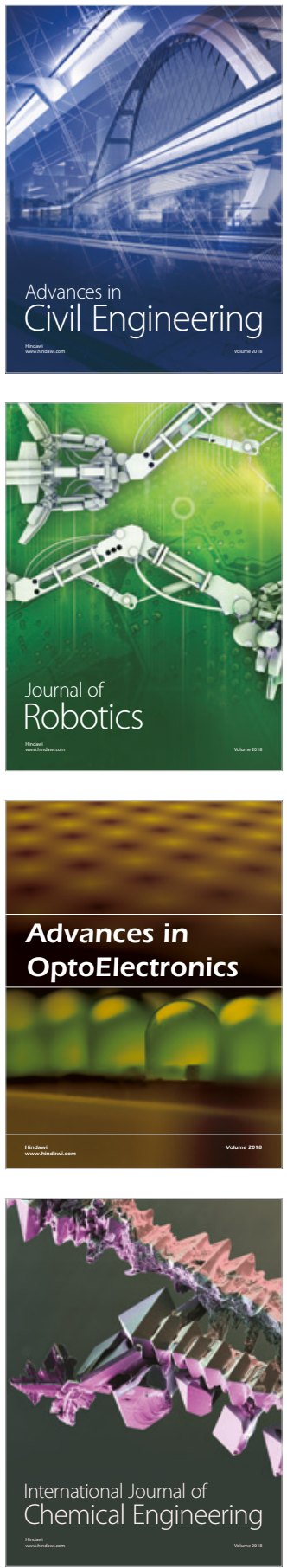

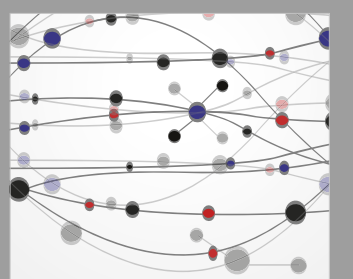

\section{Rotating \\ Machinery}

The Scientific World Journal

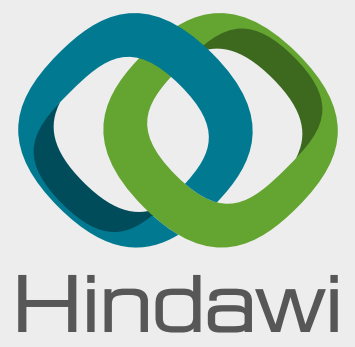

Submit your manuscripts at

www.hindawi.com
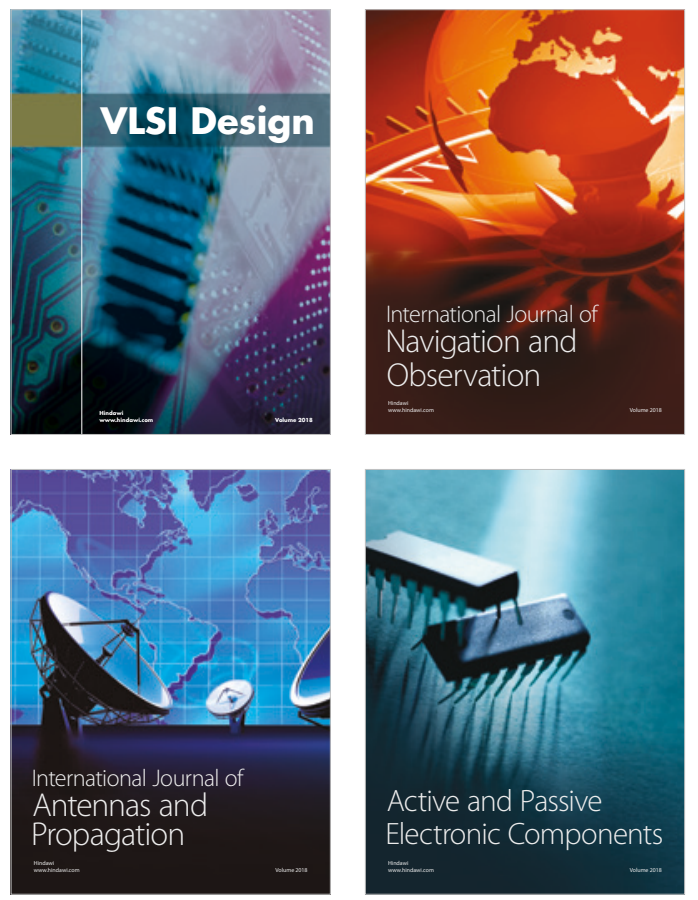
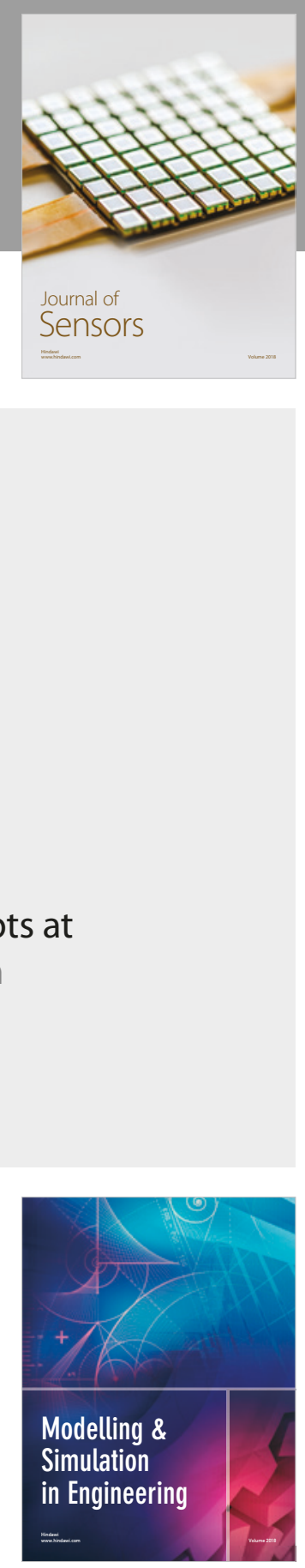

\section{Advances \\ Multimedia}
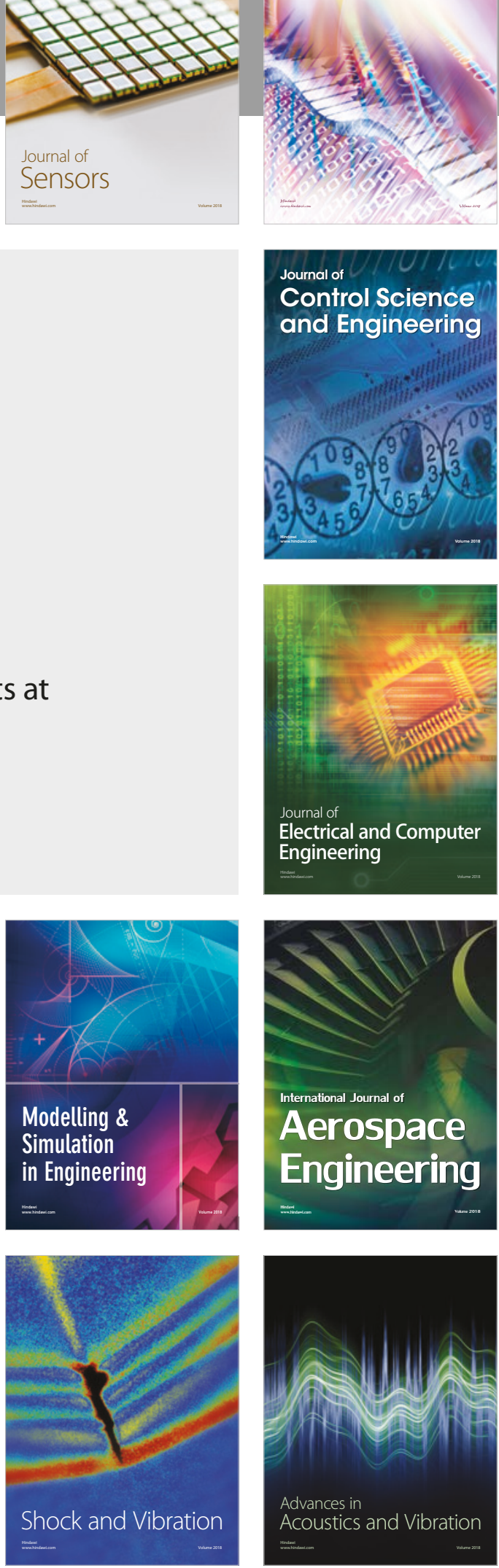